\title{
Diagnostic problems in acquired bone marrow failure syndromes
}

\author{
Shinji Nakao ${ }^{1}$
}

Received: 20 June 2016 / Revised: 28 June 2016 / Accepted: 28 June 2016 / Published online: 5 July 2016

(C) The Japanese Society of Hematology 2016

\section{Introduction}

Acquired bone marrow (BM) failure syndromes are heterogeneous diseases characterized by cytopenias caused by a decrease in or malfunction of hematopoietic stem/progenitor cells (HSPCs). These syndromes include acquired aplastic anemia (AA), myelodysplastic syndromes (MDS), paroxysmal nocturnal hemoglobinuria $(\mathrm{PNH})$, and acquired amegakaryocytic thrombocytopenia. Differential diagnosis can be difficult as these syndromes are primarily defined by cell morphology. Hemolytic PNH might at first seem easily distinguishable from other forms of bone marrow failure, since patients with PNH present with unique symptoms and laboratory findings, such as hemoglobinuria and a marked increase in the serum LDH levels. In reality, however, even experienced hematologists often misdiagnose PNH with moderate hemolysis of MDS. This is because PNH patients with BM failure exhibit significant signs of dysplasia in immature BM cells, which cannot be discriminated from MDS by experienced pathologists [1]. Misdiagnosis leads to inappropriate management of $\mathrm{PNH}$ patients, such as red blood cell transfusions alone without eculizumab therapy and allogenic stem cell transplantation from unrelated donors. Some patients may be left untreated until they develop life-threatening thromboembolism events, such as cerebral infarction.

Shinji Nakao

snakao8205@staff.kanazawa-u.ac.jp

1 Department of Hematology/Pulmonary Medicine, Institute of Medical, Pharmaceutical, and Health Sciences, Kanazawa University, Kanazawa, Japan

\section{How do dysplastic signs develop in patients with BM failure-type PNH and PNHsc?}

The dysplastic signs in PNH patients can be ascribed to co-existing MDS clones, which over time can give rise to acute myeloid leukemia. Abnormal HSPC clones may be present and exhibit dysplastic signs at the diagnosis of BM failure-type PNH or subclinical PNH (PNHsc). However, PNH-phenotype cells are rarely detected in patients in bona fide preleukemic states, such as RARS and RAEB [2]. A recent genomic analysis of hemolytic PNH patients revealed a low incidence of genomic abnormalities associated with MDS [3]. A genomic analysis of a large number of AA patients recently published by our group revealed a lower frequency of abnormalities in MDS-related genes, such as TP53 and RUNX1, in AA patients with PIGA mutations than in patients without $P I G A$ mutations [4]. It is thus unlikely that the dysplastic signs in PNH patients represent co-existing abnormal MDS clones.

Dysplastic signs in immature blood cells can be caused by extrinsic factors, such as nutritional deficiencies and toxins. Some patients with collagen diseases show dysplastic signs of immature cells similar to those of patients with MDS. In patients with AA characterized by small populations of PNH-type cells, dysplastic features typically disappear after they achieve hematologic remission following immunosuppressive therapy [5]. Reversible dysplasia, such as occurs in inflammatory diseases and immune-mediated BM failure, suggests that inflammatory cytokines produced locally in the BM may induce dysplastic changes in immature blood cells. It is thus important to bear in mind that the immune pathophysiology of BM failure, which allows PIGA-mutant HSPCs to contribute to hematopoiesis, is often associated with some degree of erythroid and granulocytic dysplasia. 


\section{Can we distinguish AA from MDS using the current diagnostic criteria?}

A more common and serious problem than the confusion between PNH and MDS is the misdiagnosis of benign immune-mediated BM failure as MDS. This happens because dysplasia is commonly seen in the BM of AA patients with small populations of PNH-type cells, just like in the BM of PNH patients. In such cases, the BM is not consistently hypocellular, due to residual hot spots [6]. Although the detection of a hypocellular BM is a prerequisite for diagnosing $\mathrm{AA}$, assessing $\mathrm{BM}$ cellularity in patients with BM failure can be difficult, particularly when the cytopenia is not severe. Even when the BM of a given bone site is grossly replaced with fat tissue as a result of the immune-mediated destruction of HSPCs, some hematopoietic nests may remain in other bone sites and may show hypercellularity due to increased BM activity that compensates for the decreased hematopoiesis [7]. BM aspiration or biopsies of these hot spots may produce erroneous results. When pathological reports of BM examinations show hyper- or normal cellularity, the attending physician does not generally consider the differential diagnosis of AA. As a result of the confusion between AA and low-risk MDS, many patients with immune-mediated BM failure are being treated inappropriately, such as with azacitidine and stem cell transplantation from unrelated donors.

\section{Practical approach to treatments of acquired BM failure syndromes}

The Progress in Hematology series in this issue includes three important articles on the diagnosis and treatment of BM failure syndromes. The most important issue in managing patients with $\mathrm{BM}$ failure syndromes is not determining the diagnosis, but treating the patients in the optimal way appropriate to the underlying pathophysiology. Figure 1 shows a concept of autoimmune hematopoietic failure (AIHM), which is common across the four different subsets of BM failure. Although each disease has a unique manifestation, the immune mechanisms shown in blue are shared by all four diseases. When a patient with BM failure possesses a marker of immune pathophysiology such as PNH-type cells [8] or HLA allele-lacking cells [9], the patient should be treated with immunosuppressive therapy, irrespective of the formal diagnosis.
Acquired aplastic anemia

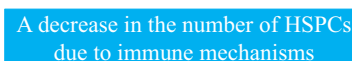

Acquired amegakaryocytic thrombocytopenia PNH with bone marrow
failure

Low-risk MDS

Genetic abnormalities in HSPCs

Fig. 1 Concept of autoimmune hematopoietic failure (AIHM). The blue rectangle represents a subset of bone marrow (BM) failure due to an immune-mediated reduction in the numbers of hematopoietic stem/progenitor cells (HSPCs). The yellow rectangle represents another subset of BM failure based on genetic abnormalities in HSPCs

\section{References}

1. Araten DJ, Swirsky D, Karadimitris A, Notaro R, Nafa K, Bessler $\mathrm{M}$, et al. Cytogenetic and morphological abnormalities in paroxysmal nocturnal haemoglobinuria. Br J Haematol. 2001;115:360-8.

2. Sugimori C, Mochizuki K, Qi Z, Sugimori N, Ishiyama K, Kondo Y, et al. Origin and fate of blood cells deficient in glycosylphosphatidylinositol-anchored protein among patients with bone marrow failure. Br J Haematol. 2009;147:102-12.

3. Shen W, Clemente MJ, Hosono N, Yoshida K, Przychodzen B, Yoshizato T, et al. Deep sequencing reveals stepwise mutation acquisition in paroxysmal nocturnal hemoglobinuria. J Clin Invest. 2014;124:4529-38.

4. Yoshizato T, Dumitriu B, Hosokawa K, Makishima H, Yoshida $\mathrm{K}$, Townsley D, et al. Somatic mutations and clonal hematopoiesis in aplastic anemia. N Engl J Med. 2015;373:35-47.

5. Nakao S, Sugimori C, Yamazaki H. Clinical significance of a small population of paroxysmal nocturnal hemoglobinuria-type cells in the management of bone marrow failure. Int J Hematol. 2006;84:118-22.

6. Yamazaki H, Nakao S. Border between aplastic anemia and myelodysplastic syndrome. Int J Hematol. 2013;97:558-63.

7. Nishimura R, Mase S, Araki R, Fujiki T, Kuroda R, Maeba H, et al. Massive hyper-reactive hematopoietic nests in bilateral iliac bones in a patient with mild aplastic anemia. Pediatr Blood Cancer. 2014;61:1903-4.

8. Kulagin A, Lisukov I, Ivanova M, Golubovskaya I, Kruchkova I, Bondarenko S, et al. Prognostic value of paroxysmal nocturnal haemoglobinuria clone presence in aplastic anaemia patients treated with combined immunosuppression: results of two-centre prospective study. Br J Haematol. 2014;164:546-54.

9. Katagiri T, Sato-Otsubo A, Kashiwase K, Morishima S, Sato Y, Mori Y, et al. Frequent loss of HLA alleles associated with copy number-neutral $6 \mathrm{pLOH}$ in acquired aplastic anemia. Blood. 2011;118:6601-9. 